\title{
Extraction and Antioxidant Activity of Flavones from Salvia miltiorrhiza
}

\author{
Guangtao Jia ${ }^{1, *}$ \\ School of Biology Science and Techology, Weifang Medical University, Weifang, China \\ *Corresponding author, e-mail:guangtaojia@163.com
}

\begin{abstract}
Keywords: Salvia miltiorrhiza; flavones; Ultrasonic extraction; antioxidation activity
Abstract: 1. Objective: Salvia miltiorrhiza is a traditional Chinese herb medicine and is widely used in clinic application. This study is intended to extract flavones from Salvia miltiorrhiza by ultrasonic method in order to evaluate its antioxidation activity. 2. Methods: (1) Spectrophotometry is used to determine content of flavones from Salvia miltiorrhiza; (2) Ultrasonic method was used to extract flavones from Salvia miltiorrhiza and $80 \%$ ethanol-water as the solution. The effect of the different volume of solvent, extraction time and frequency were conducted (3) The antioxidation of Salvia miltiorrhiza flavones is evaluated by pyrogallol autoxidation and deoxyribose degradation. 3. Results: (1) Phenol - sulfuric acid method was established to test the content of flavones from Salvia miltiorrhiza. The results were in good linear relationship, and $\mathrm{y}=0.0119 \mathrm{x}-0.0061, \mathrm{R}^{2}=$ 0.9996. (2) The extraction process of Salvia miltiorrhiza flavones by ultrasonic method was $5 \mathrm{~g}$ raw material, $245 \mathrm{~mL} 80 \%$ ethanol-water solution, twice extraction, $40 \mathrm{~min}$ for each. (3) Antioxidtion tests indicated that with the concentration $0.05 \mathrm{mg} / \mathrm{mL}$ of flavones, the inhibition rate for superoxide anion radical could reach $76.89 \%$ and $58.54 \%$ for hydroxyl radical, respectively. And the reducing power was likely to increase with the increasing of flavones concentration. 4. Conclusion: Ultrasonic method could effectively extract flavones from Salvia miltiorrhiza. Antioxidation tests indicate that flavones from Salvia miltiorrhiza have a certain removal effect on superoxide anion radical and hydroxyl radical.
\end{abstract}

\section{INTRODUCTION}

Salvia miltiorrhiza is a traditional Chinese herb medicine. It is mainly used in treatment of stroke, cardio-vascular disease, hyperlipidemia, and cerebro-vascular disease, etc ${ }^{[1-3]}$. At present, studies toward Salvia miltiorrhiza activity are mainly concentrated on salvianolic acid, tanshinones and caffeic acid and their pharmacology function ${ }^{[4]}$. Flavones compound is a significant bioactive substance which can enhance immune function for antitumor, anticoagulation, blood fat regulation, anti-aging, anti-virus, etc ${ }^{[5,6]}$. The biological function of flavones is closely related to cleaning power of active oxygen radicals. Therefore, flavones gained a large scale of popularity. However, fewer studies are on flavones from Salvia miltiorrhiza which is $12 \% \sim 38 \%$ in it ${ }^{[7]}$.

This study is intended to extract flavones from Salvia miltiorrhiza by ultrasonic method and evaluate their antioxiditon activity, in order to provide reliable data and information for further development of Salvia miltiorrhiza resources.

\section{MATERIALS AND METHODS}

\section{A. Maiterials}

\section{1)Herb Medicine}

Salvia miltiorrhiza purchased from Beijing Tong Ren Tang Group Co., Ltd., was identified by Chinese medicine teaching and research section of Weifang Medical University.

2) Reagents

Rutin (purity $\geq 98.5 \%$ ) purchased from Sigma was used as the reference substance to quantify the flavones extracted from Salvia miltiorrhiza.

Ethanol (95\%), hydrochloric acid, sulfuric acid, FeSO 4 -EDTA (10 mmol/L), 2-deoxyribose $(10 \mathrm{mmol} / \mathrm{L}) 、$ phosphate buffer $(\mathrm{pH}=7.4,0.1 \mathrm{~mol} / \mathrm{L}), \mathrm{H}_{2} \mathrm{O}_{2}(10 \mathrm{mmol} / \mathrm{L}), \mathrm{TCA}(2.8 \%), \quad$ TBA $(1.0 \%)$, phenol $(6 \%$ and $80 \%)$, distilled water, chloroform, n-butyl alcohol、 Tris-HCl buffer $(\mathrm{pH}=8.2)$, pyrogallic acid $(45 \mathrm{mmol} / \mathrm{L}$ pyrogallol soluted in $10 \mathrm{mmol} / \mathrm{L} \mathrm{HC1})$, vitamin $\mathrm{C}(0.024 \mathrm{mg} / \mathrm{mL})$, all the regents used in this study were analytical pure. 


\section{B. Methods}

\section{1)Phenol-sulfuric acid method for determining flavones content}

a) Preparation of flavones samples: $5 \mathrm{~g}$ Salvia miltiorrhiza powders mixed with $80 \%$ ethanol-water $250 \mathrm{~mL}$ in proportion by ultrasonic extraction. Then centrifugal separation method was used to obtain supernatant and the residue drying was performed to gain crude flavones from Salvia miltiorrhiza. The crude flavones dissolved into $80 \%$ ethanol-water to gain sample of flavone solution.

b) Standard curve for flavones: Rutin (100mg) was dissolved into $80 \%$ ethanol-water $(250 \mathrm{~mL})$ to gain standard solution. Different volumes of standard solution $(0.0,0.2,0.6,1.0,1.4$ and $1.8 \mathrm{~mL})$ were put into 6 tubes, and distilled water was added to $2.0 \mathrm{~mL}$. Then $\mathrm{NaNO}_{2}(5 \%$, $0.3 \mathrm{~mL})$ and $\mathrm{Al}\left(\mathrm{NO}_{3}\right)_{3}(10 \%, 0.3 \mathrm{~mL})$ were added into each tube by order, and standing in room temperature for $6 \mathrm{~min}$. $4 \mathrm{~mL} \mathrm{NaOH}(1 \mathrm{~mol} / \mathrm{L})$ was added into the tube at last and standing for $15 \mathrm{~min}$ after shaked up. The solutions were tested with an ultraviolet spectrophotometer (Shanghai Meipuda Instrument Co., LTD) at 510nm. 80\% ethanol-water $(1 \mathrm{~mL})$ as the blank control solution was prepared with the method mentioned above. Taking the absorbance A as the longitudinal coordinate and the glucose concentration $\mathrm{C}$ as the horizontal coordinate, the regression equation was obtained, and the standard curve was established.

c) Determination of flavones content from Salvia miltiorrhiza: Crude drying flavones products $0.05 \mathrm{~g}$ dissolved into distilled water $500 \mathrm{~mL}$ to obtain the sample solution, and then treated with the method mentioned in B. a) The absorbance was recorded with ultraviolet spectrophotometer to calculate the content of flavones :

$\mathrm{Y}(\%)=\mathrm{M} / \mathrm{W} \times 100 \%$

Where $\mathrm{Y}$ is the yield ratio of flavones $(\%) ; \mathrm{M}$ is the measured quantity of crude flavones $(\mathrm{g}) ; \mathrm{W}$ is original weigh of Salvia miltiorrhiza powders.

\section{2)Ultrasonic extraction of flavones}

Effect of different volumes of solvent, extraction time and extraction frequency was test by ultrasonic extraction. Crude flavones were prepared by the method mentioned yin B. a). The yield ratio of flavones with three factors was calculated, respectively

\section{3) Determination of in vitro antioxidant capacity of Salvia miltiorrhiza flavones}

a)Determination of superoxide anion radical scavenging capacity: $9 \mathrm{~mL}$ Tris- $\mathrm{HCl}$ buffer $(\mathrm{pH}=8.2)$ was added into a tube, insulation for $20 \mathrm{~min}$ in a water bath thermal; then $0.5 \mathrm{~mL}$ preheated $\left(25^{\circ} \mathrm{C}\right)$ pyrogallic acid was mixed with the solution above, and poured into colorimetric tube immediately. After $3 \mathrm{~min}$, vitamin $\mathrm{C}(0.5 \mathrm{~mL})$ was added into the colorimetric tube and kept for $5 \mathrm{~min}$ in room temperature. The absorbance $\mathrm{D}_{0}$ was recorded at $420 \mathrm{~nm}$, which was the autoxidation rate of pyrogallic acid. And the final volume of the reaction system was $10 \mathrm{~mL}$.

Sample solution of crude flavones was added into Tris-HCl buffer solution, and the sample process mentioned above was performed, then the absorbance $\mathrm{D}_{1}$ was recorded at $420 \mathrm{~nm}$. Sample solution of crude flavones was replaced by distilled water to be the blank control $\left(\mathrm{D}_{2}\right)$. The following equation was to quantify the inhibition rate of superoxide anion radical:

$$
\text { Inhibition rate }(\%)=\frac{D_{0}-\left(D_{1}-D_{2}\right)}{D_{0} \times 100} \times 100 \%
$$

b) Determination of hydroxyl radical scavenging capacity: $\mathrm{FeSO}_{4}$-EDTA mixture $(0.2 \mathrm{~mL})$ and 2-deoxyribose $(0.5 \mathrm{~mL})$ were mixed into a tube; then different concentration of sample solution of crude flavones $(0.5 \mathrm{~mL})$ was added into the tube; $\mathrm{H}_{2} \mathrm{O}_{2}(0.2 \mathrm{~mL})$ was put into the tube after using phosphate buffer to get the volume to $1.8 \mathrm{~mL}$; and the tube insulation for $20 \mathrm{~min}$ in a water bath thermal. Furthermore, TCA $(1.0 \mathrm{~mL})$ and TBA $(1.0 \mathrm{~mL})$ were mixed and put into the tube, then placed into boiling water bath for $15 \mathrm{~min}$. At last, the absorbance $\left(\mathrm{A}_{2}\right)$ at $532 \mathrm{~nm}$ was recorded after the tube was cool down. Sample solution of crude flavones was replaced by distilled water to be the blank control $\left(\mathrm{A}_{1}\right)$. The following equation was to quantify the inhibition rate of superoxide anion radical: 
Inhibition rate $(\%)=\left(\mathrm{A}_{1}-\mathrm{A}_{2}\right) / \mathrm{A}_{1} \times 100 \%$

\section{RESULTS}

\section{A. Establishment of quantitative standard curve of Salvia miltiorrhiza flavones}

With different concentration of standard glucose solution added with phenol-sulfuric acid, absorbance was determined at $490 \mathrm{~nm}$ for establishment of quantitative standard curve of Salvia miltiorrhiza (Fig. 1). With fitting, calculation formula of Salvia miltiorrhiza flavones was as follow $\mathrm{y}=0.0119 \mathrm{x}-0.0061$. A good linear relationship was shown in Fig 1 , with $\mathrm{R}^{2}=0.9996$, which can satisfy the needs for determination of Salvia miltiorrhiza flavones.

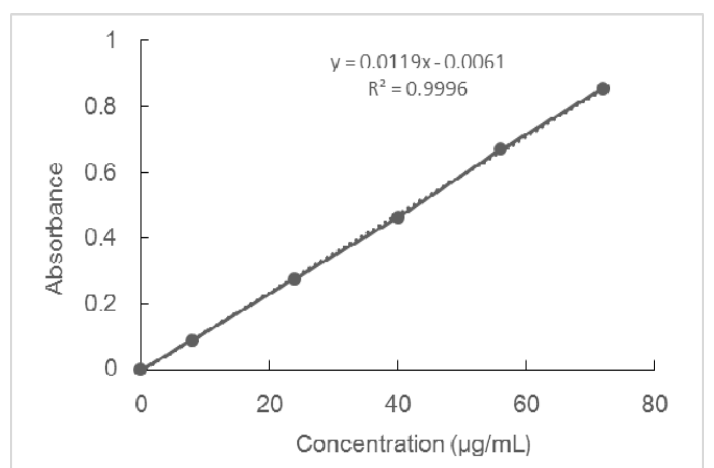

Fig. 1 Quantitative standard curve of flavones from Salvia miltiorrhiza

\section{B. Process of Salvia Miltiorrhize Flavones Extraction by Ultrasonic Waves}

1) Effect on flavones extraction yield by extraction ratio of solid to liquid: With fixed amount of Salvia miltiorrhiza and different quantity of $80 \%$ ethanol-water solution for ultrasonic extraction, the results are shown in Fig. 2. When the extraction ratio of solid to liquid was $1: 45(\mathrm{~g}: \mathrm{mL})$, the extraction capacity was the highest. With an increasing amount of solution appliance, the concentration of flavones was slightly rather than obviously increasing too. Therefore, $5 \mathrm{~g}$ Salvia miltiorrhiza mixed with $245 \mathrm{~mL}$ ethanol-water $(80 \%)$ to extraction flavones.

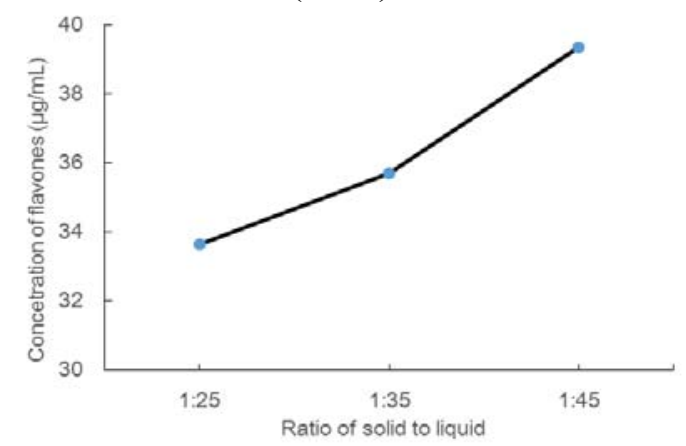

Fig.2 Effect on extraction quantity by ratio of solid to liquid

2) Effect on flavones extraction yield by extraction time

Effect on flavones extraction in different time duration can be seen in Fig. 3. The results indicate that the extraction capacity is obviously increased with time goes by. When the extraction time is $40 \mathrm{~min}$, the concentration of flavones is $33.66 \mu \mathrm{g} / \mathrm{mL}$, which is similar to the result of extraction time 50min. From the aspect of efficiency, 40min was selected as extraction time. 


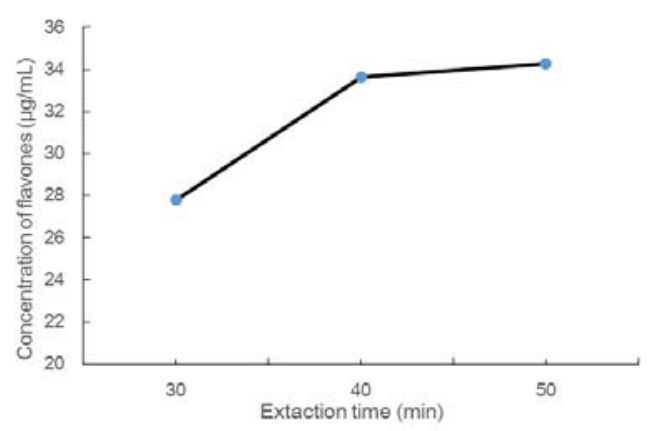

Fig.3 Effect on flavones extraction yield by extraction time

\section{3) Effect on flavones extraction yield by extraction frequency}

Effects are shown in Fig. 4 about frequent extraction in solutions with one portion of Salvia miltiorrhiza powder. The results indicated that with an increasing frequency of extraction, the extraction capacity was likely to increase. For the second time extraction, the concentration of flavones was $55.35 \mu \mathrm{g} / \mathrm{mL}$, while the data was $69.86 \mu \mathrm{g} / \mathrm{mL}$ in the third time extraction. With a slight difference in between, and for economical purpose, two times extraction was recognized as the best frequency of extraction.

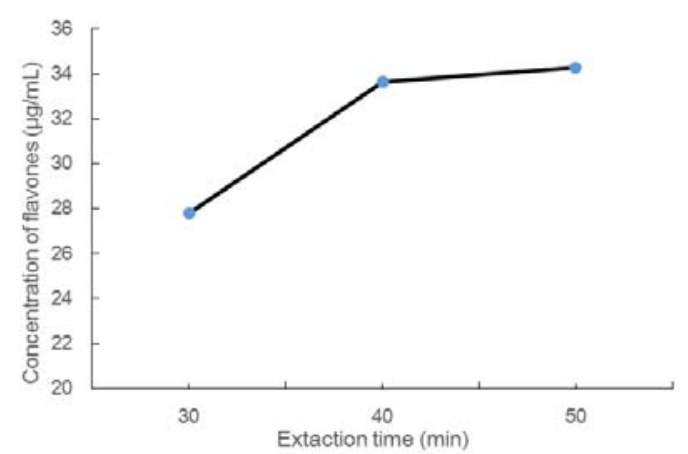

Fig.4 Effect on flavones yield by extraction frequency

\section{Results for Flavones Antioxidant Activity Determination}

1) Ability to remove superoxide anion

Different samples, with reagents added in, were determined by spectrophotometer for absorbance. Three tests were applied on each sample before calculating their cleaning power for superoxide anion radicals by the flavones under different concentrations based on formulate (2). The results can be seen in Fig. 5. The results indicated that flavones of Salvia miltiorrhiza had certain cleaning power to superoxide anion radicals. When the concentration of flavones solution was $0.01 \mathrm{mg} / \mathrm{mL}$, the inhibition rate was $76.89 \%$. In addition, the inhibition rate was increasing constantly with the increasing of flavones concentration within the above range.

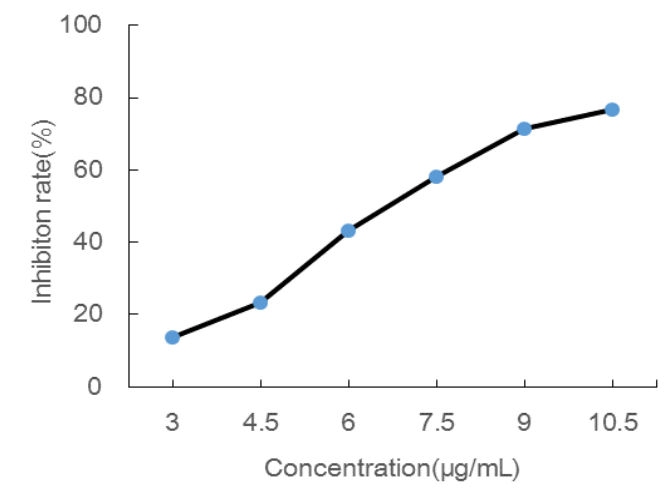

Fig.5 Ability of Flavones to remove superoxide anion radicals

2) Ability to remove hydroxyl radicals

Different samples, with reagents added in, were determined by spectrophotometer for absorbance. Three tests were applied on each sample before calculating their cleaning power for hydroxyl radicals by the flavones under different concentrations based on formulate (3). The results 
can be seen in Fig. 6. The results indicated that flavones of Salvia miltiorrhiza had certain cleaning power to hydroxyl radicals. When the concentration of flavones solution was $0.01 \mathrm{mg} / \mathrm{mL}$, the inhibition rate was $58.54 \%$. In addition, the inhibition rate was increasing constantly with the increasing of flavones concentration within the above range.

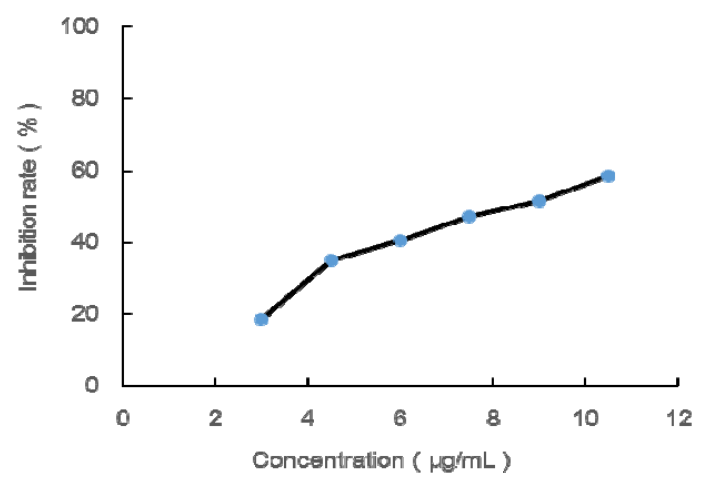

Fig. 6 Ability of Flavones to remove hydroxyl radicals

\section{CONCLUSION}

This research adopted ultrasonic extraction for flavones in Salvia miltiorrhiza. Rutin was taken as the reference for the establishment of quantitative standard curve of Salvia miltiorrhiza flavones by adopting phenol-sulfuric acid colorimetry. The results were in good linear relationship, and can meet the quantitative requirements for crude flavones.

When ultrasonic was used for flavones extraction, the extraction capacity slightly increased while the solution was increased. Since the extraction capacity did not change much, this result was possibly because of the increased solution quantity and processing amount. The best extraction ratio of solid to liquid was 1:45 (g:mL). The extraction capacity increased with the extraction time extended, and from the aspect of efficiency, 40min was selected as the extraction time. The extraction capacity was promoted prominently for the first and second extraction, while the concentration of flavones was increasing slowly to a fixed number for the third time extraction. So from the economical and practical aspects, two times of extraction was taken as the most ideal frequency.

To certain extent, Salvia miltiorrhiza flavones were useful to clean superoxide anion radicals and hydroxyl radicals. The cleaning power was increased with the increased concentration of flavones.

\section{Acknowledgements}

Supported by a project grant from the Foundation for Outstanding Young Scientist in Shandong Province (Grand No. BS2013YY063).

\section{References}

[1] X. C. Ge and J. Y. Wu. Tanshinone production and isoprenoid pathways in Salvia miltiorrhiza hairy roots induced by $\mathrm{Ag}^{+}$and yeast elicitor. Plant Science, 2005, 168: 487-491.

[2] H. Wang, Q. Wang, H. M. Luo, et al. Quantitative analysis of Salvia miltiorrhiza flavones and determination of its composition by HPCE. Chinese Archives of Traditional Chinese Medicine, 2007, 25(4): 827-829.

[3] T. Cheng. Cardiovascular effects of Danshen. International Journal of Cardiology, 2007, 121(4): 9-22.

[4] R. W. Jiang, K. M. Lau, P. M. Hon, et al. Chemistry and biological activities of caffeic acid derivatives from Salvia miltiorrhiza. Current Medical Chemistry, 2005, 12(2): 237-246. 
[5] M. Y. Xie and S. P. Nie. A review about the research of structure and function of flavones from natural products. Journal of Chinese Institute of Food Science and Technology, 2010, 10(2): $1-10$.

[6] L. H. Shen, J. S. Wang, Y. Li, et al. Research and application of plant flavones. Chinese Agricultural Science Bulletin, 2011, 27(02): 349-352.

[7] X. Y. Wei, M. B. Jing, J. C. Wang, et al. Preliminary study on Salvia miltiorrhiza bung endophytic fungus. Academic Journal of Xi' an Jiaotong University, 2010, 22(4): 241-246. 